\title{
Étude \\ de la protection contre les crues dans le bassin de l'Orb
}

\author{
par F. Brun et G. Dumas
}

Agence de Bassin Rhône-Méditerranée-Corse

Le but de cette étude, dont l'exécution fut confiée en 1967 à l'Agence de Bassin Rhône-Méditerranée-Corse par le Ministère de l'Equipement et du Logement (Service Central Hydrologique et Service Hydrologique Centralisateur du Bassin Méditerranéen), a été d'apporter des éléments pour la mise au point d'un plan général de protection contre les crues dans le bassin de l'Orb, fleuve côtier du Languedoc.

Ce cours d'eau, en effet, comme tous les cours d'eau de type cévenol, donne lieu à des crues brutales qui s'accompagnent souvent de dégâts importants et parfois, comme on le verra ci-après, de pertes de vies humaines.

Mais l'intérêt de l'étude - et c'est à ce titre qu'elle nous paraît mériter d'être présentée ici - était aussi d'ordre méthodologique: il s'agissait, en particulier, de mettre en application les nouvelles méthodes de calcul économique en matière de protection contre les crues qui venaient d'être mises au point, sous l'égide du Service Central Hydrologique du Ministère de l'Equipement, par le B.C.E.O.M. (Bureau Central d'Etudes pour les Equipements d'Outre-Mer) à l'occasion d'études intéressant le bassin parisien et le bassin de l'Aude $\left.{ }^{a}\right)$.

Le schéma de l'étude, qui pourrait s'appliquer à tout autre problème analogue, a été le suivant:

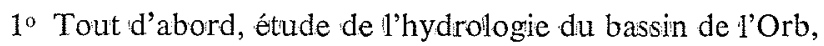
en vue de déterminer l'importance des crues, en débits et en volumes, en fonction de leur fréquence, et les hauteurs, surfaces et durées d'inondation correspondantes.

$2^{\circ}$ Exécution d'une étude économique du coût des crues, menée à partir d'observations et d'enquêtes faites à l'occasion de deux crues récentes, celles de décembre 1953 et

(a) Le principe de ces méthodes a du reste fait l'objet d'un exposé du B.C.E.O.M. devant la Société Hydrotechnique de France à l'occasion des Dixièmes Joumées de l'Hydraulique. en 1968. d'ootobre 1969, qui a permis d'établir la relation liant le coût des dommages dus aux crues à l'importance et à la fréquence de celles-ci. Ces résultats permettent de déduire, par intégration et actualisation à l'année zéro, la valeur actuelle des dommages dus aux crues susceptibles de se produire dans l'avenir, à la fois dans les conditions hydrauliques existantes et dans toutes autres conditions hydrauliques résultant d'aménagement à créer;

$3^{\circ}$ La troisième partie de l'étude a consisté justement à examiner la possibilité et l'intérêt de différents types d'aménagements susceptibles d'améliorer les conditions d'écoullements des crues et d'en diminuer ainsi l'ampleur génératrice de dommages. A partir des études hydrologiques et économiques précédentes, on a essayé d'associer à chacune des hypothèses d'aménagement étudiées le nouveau coût actualisé des dommages des crues futures ainsi atténuées: par rapprochement avec le coût analogue calculé en l'absence de tout aménagement, et compte tenu des coûts d'investissement et d'exploitation des ouvrages à prévoir, on a pu alors établir une comparaison économique - sommaire étant donné le degré de précision des études faites - entre les différents aménagements considérés.

\section{L'étude hydrologique}

\section{L’Orb et son Bassin versant}

L'Orb prend sa source dans le sud du Causse du Larzac, à $825 \mathrm{~m}$ d'altitude. Il reçoit ( $)$, en rive droite, la Mare, le Jaur, le Rieuberlou, le Vernazobre et le Lirou, en rive

(b) Voir carte générale du bassin (fig. $\mathrm{n}^{\circ} 1$ ). 
gauche, le Rieutord et le Taunou, arrose principalement Bédarieux, Lamalou-les-Bains, Cessenon, Béziers, Sérignan et, après un parcours total de quelque $140 \mathrm{~km}$ rejoint la Méditerranée à Valras où la superficie de son bassin versant est de $1540 \mathrm{~km}^{2}$. Sur les deux premiers tiers de son cours, jusque vers Cessenon, l'Orb coule en zone montagneuse et accidentée, de substratum essentiellement schisteux; la pente moyenne de ce tronçon dépasse $8 \mathrm{~m} / \mathrm{km}$. Puis l'Orb rentre en plaine, et la pente moyenne des derniers $40 \mathrm{~km}$ de son cours n'est plus que de $1 \mathrm{~m} / \mathrm{km}$ environ. A l'approche de la mer, dan's la basse plaine, là où les inondations s'étalent, la pente du cours d'eau devient très faible.

La pluviométrie moyenne annuelle s'étage de $1500 \mathrm{~mm}$ sur le haut bassin à $600 \mathrm{~mm}$ au bord de la mer.
Le régime de $\mathbb{l}^{\prime}$ Orb est un régime de type pluvial méditerranéen, influencé par la présence des Cévennes, avec des étiages accusés en été et des crues soudaines de printemps, d'automne et d'hiver.

On peut citer, à titre d'exemple de telles crues, celle du 12 septembre 1875 , de débit élevé même à Béziers, bien que causée par une pluie inférieure à $24 \mathrm{~h}$ et localisée aux affluents de la rive droite de l'Orb, qui entraîna la mort de 120 personnes à Saint-Chinian, sur le Vernazobre.

Plus près de nous, la arue de décembre 1953 intéressa tout le bassin de l'Orb et fut consécutive à une pluie atteignant $900 \mathrm{~mm}$ dans la partie supérieure du bassin, dont la moitié répartie sur cinq jours.

Indiquons ici, pour fixer les idées, que le débit moyen annuel du cours d'eau est d'environ $25 \mathrm{~m}^{3} / \mathrm{s}$ à l'embouchure.

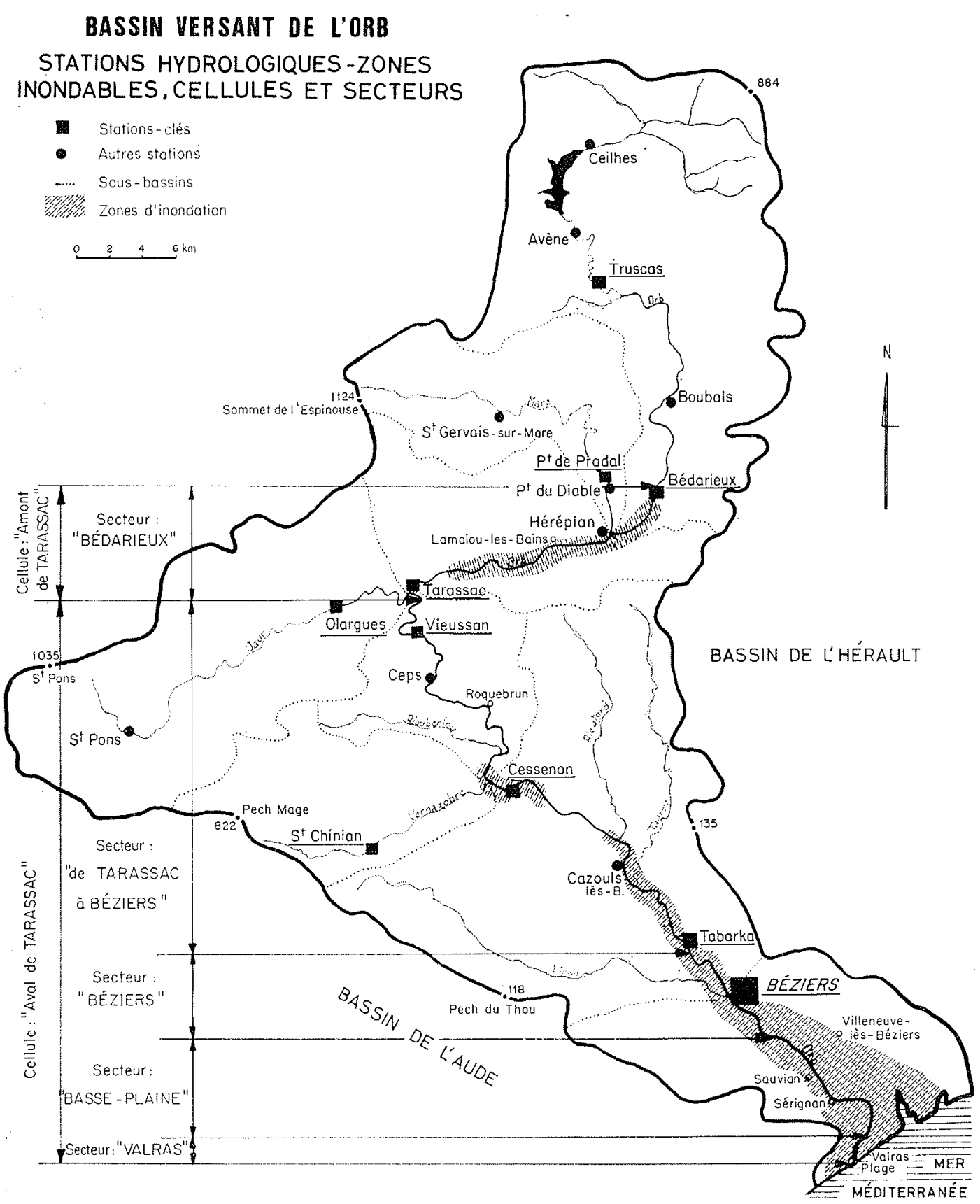




\section{Buts ef moyens de l'étude hydrologique}

Comme nous l'avons indiqué plus haut, le but de cette étude était d'aboutir à la meilleure connaissance possible des crues de l'Orb, à partir des observations disponibles, et d'établir, par extrapolation, les lois statistiques liant les paramètres représentatifs des crues - débits de pointe, volumes, hauteurs, surfaces et durées d'inondation - à leur fréquence de retour : ceci de façon à permettre, d'une part d'établir la relation liant fréquence des crues et coût des dommages correspondants, d'autre part d'étudier le fonctionnement et l'efficacité des ouvrages de protection contre les crues éventuels.

Le premier travail a consisté à rassembler le maximum de données et de mesures existantes, concernant la vingtaine de stations hydrologiques $\mathrm{du}$ bassin versant ayant fait l'objet d'une exploitation à un moment ou à un autre; certaines des séries de mesures étaient fort anciennes, pour Béziers notamment où elles remontaient jusqu'en 1842; malheureusement, la grande hétérogénéité des données, recueillies par des organismes, pour des besoins et par des procédés différents, la faible durée ou le manque de continuité des observations, ou encore le manque de stabilité des sections, n'ont permis de conserver, en première sélection, qu'une dizaine de stations - dites stations-clés (voir carte du bassin versant) - pour lesquelles des campagnes complémentaires de mesures topographiques et de jaugeages ont été lancées au cours des années 1968-1969 en collaboration avec d'autres services et organismes intervenant dans le secteur: Service de l'Equipement de l'Hérault, 5e Circonscription Electrique (devenue depuis la Circonscription Electrique Sud-Ouest) et Service du Canal du Midi, Compagnie Nationale d'Aménagement de la Région du Bas-Rhône et du Languedoc, Service Régional d'Aménagement des Eaux du Ministère de l'Agriculture..., et avec l'appui, pour les prévisions météorologiques, du Centre Météorologique Régional de Marignane. La soudaineté et la faible durée des crues, la dispersion des organismes intéressés, ont nécessité, pour l'exécution de ces campagnes, la mise au point d'un dispositif spécial de déclenchement et de coordination des interventions, avec système d'alerte, répartition des tâches entre les différentes équipes de jaugeage disponibles, échange des informations, etc. Le même dispositif était du reste utilisé pour d'autres cours d'eau de la région languedocienne.

Dans le cadre des mêmes oampagnes, il était également prévu, en cas de circonstances appropriées, l'exécution, à l'initiative du Service Hydrologique Centralisateur, d'un survol des zones inondées avec prise de photographies aériennes et lancement des enquêtes sur les dommages causés dont il sera question plus loin.

L'ensemble des données ainsi recueillies - en archives ou sur le terrain - soumises à une critique très attentive. complétées ou vérifiées le cas échéant au moyen de corrélations entre stations, de méthodes de comblement de lacunes (traitées sur ordinateur) $\left({ }^{c}\right)$, a permis, pour cinq stations hydrologiques, de tracer les courbes de tarage $\left({ }^{d}\right)$ correspondantes et d'évaluer les débits de pointe de orues pour de faibles durées de retour au moins. Il s'agit des stations suivantes, toutes situées sur l'Orb, sauf Olargues sur le Jaur: Truscas (bassin versant: $185 \mathrm{~km}^{2}$ ); Bédarieux $\left(346 \mathrm{~km}^{2}\right)$; Olargues $\left(232 \mathrm{~km}^{2}\right)$; Vieussan $\left(905 \mathrm{~km}^{2}\right)$; Béziers-Pont Rouge (aval de Béziers, $1462 \mathrm{~km}^{2}$ ). Mais ce n'est que pour les stations de Bédarieux ot de Béziers-
Pont Rouge que les durées d'observation utilisables se sont avérées suffisamment longues pour permettre un traitement statistique complet. Pour lla station de Bédarieux, en effet, on a pu retenir une série d'observations de hauteurs d'eau commençant en 1951, et pour celle de Béziers-Pont Rouge, une série commençant en 1923, auxquelles s'ajoutent un certain nombre d'observations fragmentaires ou lincomplètes plus anciennes.

\section{Principaux résultats. Notion de crues-łypes}

Crues-types. - Pour les besoins de l'étude économique, dont on traitera p'us loin, il est nécessaire d'introduire la notion de crue-type. Rappelons-en brièvement l'utilité.

Il s'agit, en effet, de pouvoir attribuer à une crue donnée une probabilité de dommages permettant de la classer dans une échelle statistique.

Cela serait aisé si le coût des dommages ne dépendait que d'un seul paramètre: hauteur maximale, ou volume de la crue, par exemple. Or, en fait, les dommages causés par une crue dépendent de nombreux paramètres: débit maximal, hauteur et durée d'inondation, vitesse de montée des eaux, volume de crue, vitesse du courant, saison, heure, etc. L'expérience montre, toutefois, que, saison et heure mises à part, ces dommages sont essentiellement fonction des hauteurs d'eau et des durées d'inondation.

On se limite donc, pour simplifier, à la prise en compte de ces deux derniers paramètres.

Par traitement statistique des limnigrammes (ou hydrogrammes) des crues observées, on dresse alors une série de courbes à deux variables: hauteur (ou débit) et durée de submersion pendant laquelle cette hauteur est dépassée, chaque courbe correspondant à des couples de valeurs ayant une périodicité de retour donnée et définissant une «crue-type » dont elle constitue le limnigramme classé. Les courbes de grande durée de retour sont obtenues, bien entendu, par extrapolation (voir fig. 2).

Pour chaque bande topographique de niveau donné de la vallée, la probabilité du coût des dommages causés par une crue-type - si elle se produisait - coût qui est supposé croître avec la durée d'inondation, est celle qui correspond à la durée de retour des couples hauteur-durée de la crue-type. On peut alors admettre qu'il en est de même pour la probabilité du coût global des dommages que causerait une telle crue-type, auquel on attribure ainsi la durée de retour correspondante.

A une crue réelle de référence, dont on a pu chiffrer les dommages, on associe alors une crue-type voisine parfois appelée "orue normalisée» — seule soumise au traitement statistique. Ce peut être la crue-type de même hauteur maximale que la crue réelle, dont on calcule les

(c) Pour cet objet, comme pour les autres prob'èmes de l'étude traités sur ordinateur, on s'est attaché à rédiger des programmes d'informatique de conception suffisamment générale pour permettre, le cas échéant, une application aisée à tous les cours d'eau du bassin Rhône-Méditerranée-Corse.

(d) A noter que les extrapolations de courbes de tarage, nécessaires pour les forts débits, ont été faites, pour plus de justesse, séparément pour les lits mineurs et les $\mathrm{va}^{\prime}$ 's d'inondation, à partir de leurs caractéristiques topographiques et hydrauliques propres. 


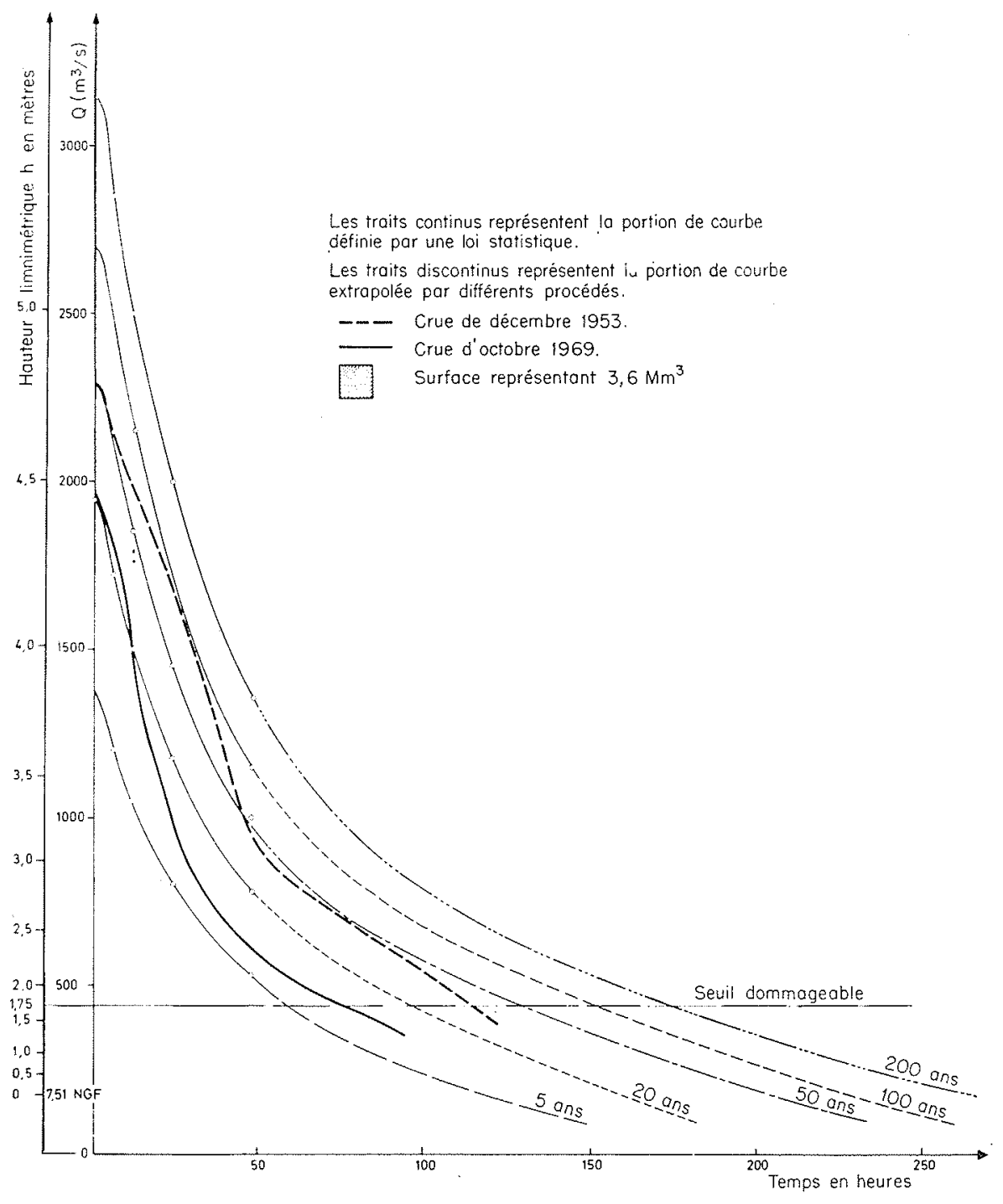

2/ Béziers Pont Rouge. Abaque des durées de retour. Crues-types; crues de 1953 el 1969.

dégâts (fictifs), un peu différents de ceux de la crue réelle, par ajustements ot approximations, moyennant certaines hypothèses sur la variation des dommages en fonction de la hauteur ou de la durée d'inondation. On peut aussi conserver la crue de référence observée - en corrigeant toutefois son coût de l'influence particulière de cortains paramètres autres que hauteur et durée: saison par exemple - et lui attribuer, de façon approchée, une probabilité de dommages (ce qui revient à lui associer la crue-type correspondant à cette probabilité) : c'est cette deuxième façon de procéder qui a été utillisée dans notre étude.

Des familles de crues-types ont été établies, par la méthode de Fréchet appliquée aux débits atteints ou dépassés pendant des durées données, pour Bédarieux et pour Béziers-Pont Rouge. La figure 2 présente les résultats pour cette dernière station; on y peut voir, par exemple, qu'une cote limnimétrique de $1,75 \mathrm{~m}$ à l'échelle, qui correspond du reste aux premiers débordements (seuil dommageable), se maintient pendant 60 heures tous les 5 ans, et 100 heures tous les 20 ans.
LES DÉBITS DE POINTE correspondant aux grandes durées de retour (jusqu'à 200 ans) ont été évalués, pour les deux stations de Bédarieux et de Béziers-Pont Rouge, par la méthode de Fréchet dans le cadre de l'étude des cruestypes (cas particulier d'une durée de dépassement nulle). On a obtenu les estimations suivantes, en $\mathrm{m}^{3} / \mathrm{s}$ :

\begin{tabular}{|c|c|c|c|c|c|}
\hline DurÉE DE RETOUR & $\begin{array}{c}5 \\
\text { ANS }\end{array}$ & $\begin{array}{c}20 \\
\text { ANS }\end{array}$ & $\begin{array}{c}50 \\
\text { ANS }\end{array}$ & $\begin{array}{c}100 \\
\text { ANS }\end{array}$ & $\begin{array}{c}200 \\
\text { ANS }\end{array}$ \\
\hline & & 775 & 1000 & 1230 & 1480 \\
\hline $\begin{array}{c}\text { Bédarieux ......... } \\
\left.\text { (B.V. }=346 \mathrm{~km}^{2}\right) \\
\text { Béziers-Pont Rouge } \\
\left(\mathrm{B} . \mathrm{V} .=1462 \mathrm{~km}^{2}\right)\end{array}$ & 1375 & 1950 & 2300 & 2700 & 3150 \\
\hline
\end{tabular}

On peut noter que lies crues les plus fortes observées tant à Bédarieux (crue de 1926 , environ $1000 \mathrm{~m}^{3} / \mathrm{s}$ ) qu'à Béziers-Pont Rouge (crule de décembre 1953, $2350 \mathrm{~m} /{ }^{3} \mathrm{~s}$ ) 


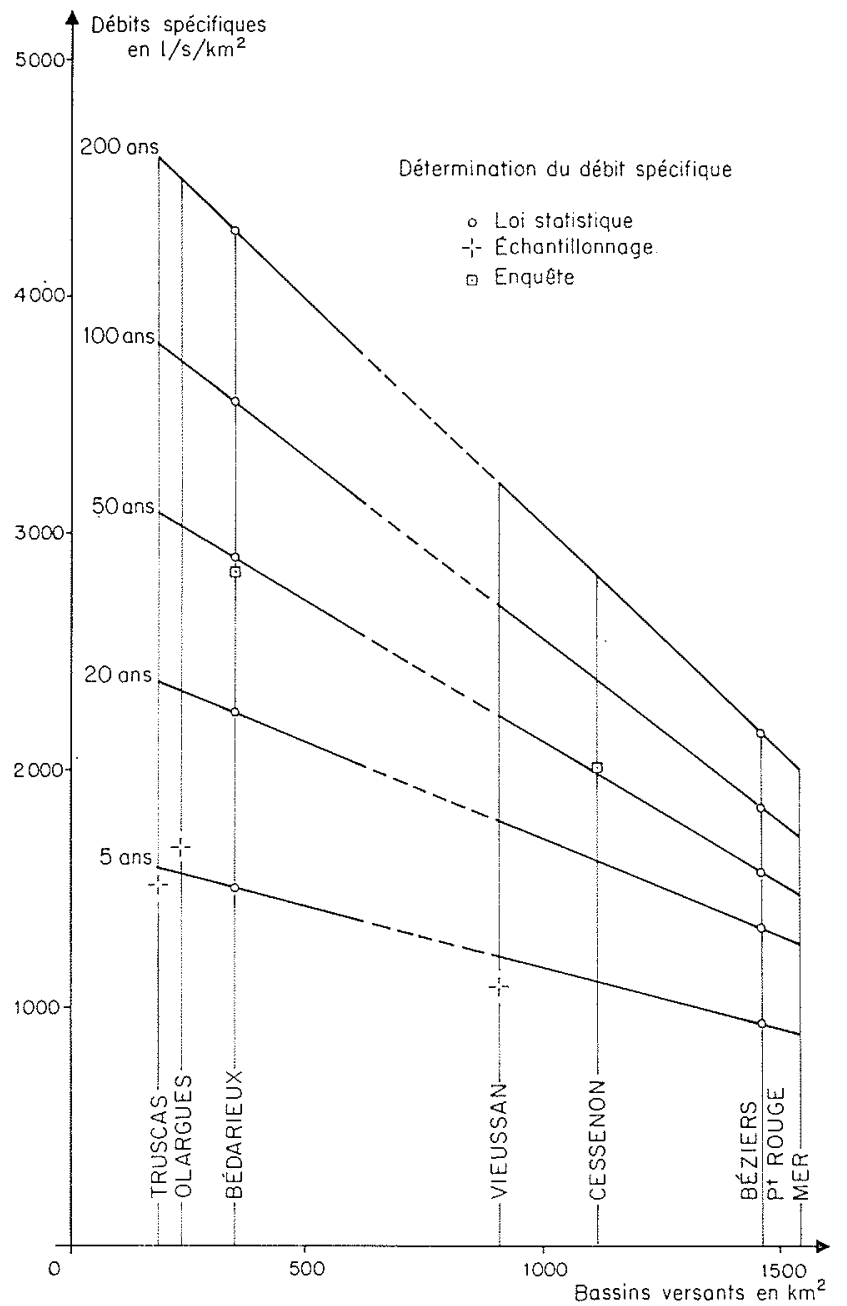

3/ Débits spécifiques de pointes de crues.
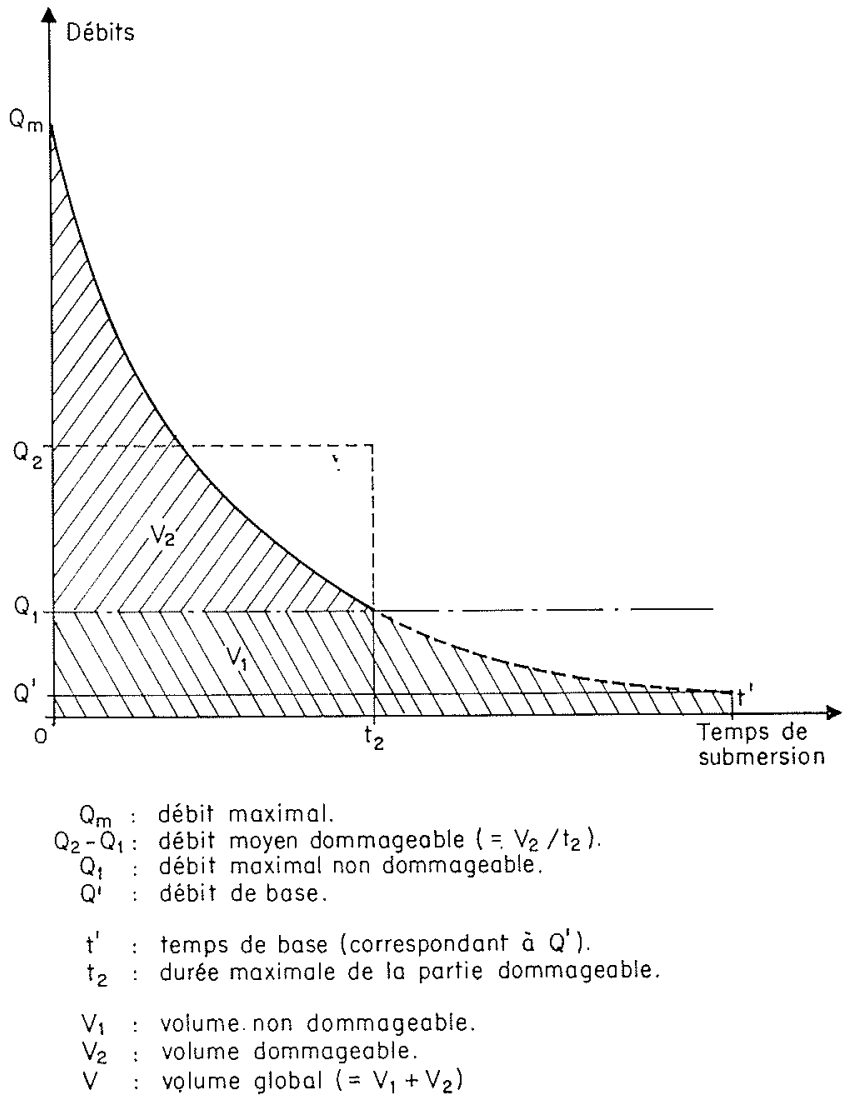

4/ Décomposition d'une crue-type.

\begin{tabular}{|c|c|c|c|c|c|c|c|c|c|c|c|}
\hline 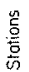 & $\begin{array}{c}T \\
\text { en añées }\end{array}$ & $\mid \begin{array}{l}\text { Partic } \\
t^{2} \text { en } \\
\text { revest }\end{array}$ & 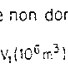 & $\begin{array}{l}\text { mogeoble } \\
v_{1} 10^{3} x^{3} / x^{2}\end{array}$ & Part & tie dom & mageable & 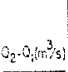 & $\begin{array}{l}\text { Résulto } \\
1\left(10^{6} \mathrm{~m}^{3}\right)\end{array}$ & $\begin{array}{l}\text { its globaux } \\
\mid v\left(1 c^{3} \mathrm{n}^{3} / \mathrm{km}^{2} \mid\right.\end{array}$ & $\frac{v_{2}}{\gamma} \times 100$ \\
\hline \multirow{5}{*}{ 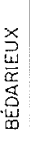 } & 5 & 100 & 44 & 127 & 30 & 15 & 44 & 139 & 59 & 171 & 25,7 \\
\hline & 20 & 162 & 72 & 209 & 47 & 36 & 104 & 213 & 108 & 313 & 33,2 \\
\hline & 50 & 208 & 103 & 299 & 73 & 55 & 159 & 209 & 158 & 458 & 34,7 \\
\hline & 100 & 271 & 153 & 443 & $145\}$ & 90 & 261 & 172 & 243 & 704 & 37,1 \\
\hline & 200 & 332 & 182 & 527 & 195 & 127 & 368 & 181 & 309 & 895 & 41,2 \\
\hline \multirow{5}{*}{ 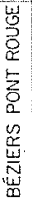 } & 5 & 144 & 170 & 116 & 59 & 76 & 52 & 358 & 246 & 168 & 30,9 \\
\hline & 20 & $18 i$ & 229 & 157 & 96 & 166 & 114 & 480 & 395 & 271 & 42 \\
\hline & 50 & 232 & 295 & 202 & 128 & 248 & 170 & 538 & 543 & 372 & 45,7 \\
\hline & 100 & 255 & 329 & 225 & $\{51$ & 332 & 227 & 610 & 661 & 452 & 50,2 \\
\hline & 200 & 295 & 381 & 261 & 173 & 432 & 296 & 693 & 813 & 557 & 53,2 \\
\hline
\end{tabular}

5/ Volumes de crues (crues-types). 


\section{L'étude économique du coût des crues}

\section{Principe}

La figure 6 rappelle le principe de l'évaluation du coût moyen des crues dans une zone donnée. On pourrait penser pouvoir utiliser, pour faire de telles évaluations, des bordereaux de coût des dommages (à l'ha cultivé; au $\mathrm{m}^{2}$ de plancher inondé) que l'on appliquerait à certaines crues de caractéristiques données. En fait, il est bien préférable de pouvoir se baser sur quelques crues de référence observées dans le passé et dont on a pu chiffrer le coût effectif des dommages.

Sur le graphique gradué en coûts et fréquences annuelles (avec durées de retour correspondantes), le report du point $\left(\mathrm{C}_{0}, \mathrm{~T}_{0}\right)$ correspondant à la crue maximale non dommageabile $\left(C_{0} \simeq 0\right)$, et des points tels que $\left(C_{1}, T_{1}\right),\left(C_{2}, T_{2}\right)$, correspondant à quelques crues-types associées à autant de crues de référence réelles étudiées, permet de tracer la courbe $C(T)$, courbe fréquentielle du coût des crues, qu'on peut considérer comme asymptotique à l'axe des ordonnées. Pour un bon tracé, il faut avoir pu évaluer les dommages d'au moins deux crues de référence de durées de retour suffisamment différentes entre elles ainsi que de $T_{0}$, durée de retour de la crue non dommageable. Bien entendu, les dommages de ces crues doivent être réévalués aux conditions économiques de l'année de l'étude, en tenant compte à la fois de la dépréciation monétaire et de l'évolution de l'économie depuis la date de la crue. Il est bon que les crues de référence ne soient pas trop anciennes, sinon cette réévaluation n'est pratiquement pas possible. L'axe des abcisses du graphique étant gradué en fréquences, ou en probabilités d'occurence, on voit qu'un simple planimétrage de la surface délimitée par la courbe $\mathrm{C}(\mathrm{T})$ et les deux axes de coordonnées fournit l'espérance mathématique du coût annuel des crues, c'est-à-dire leur coût moyen annuel $C_{m}$ (dans la situation actuelle et avant tout aménagement).

Pour des raisons de commodité pratique, on limite le planimétrage à un point tel que $\left(\mathrm{C}_{3}, \mathrm{~T}_{3}\right)$ correspondant aux inondations les plus fortes dont on puisse évaluer approximativement les dégâts et la fréquence.

\section{Application au cas de l'Orb Les enquêtes économiques}

Ces enquêtes ont été réalisées et exploitées par le B.C.E.O.M. dans le cadre de contrats passés avec le Service Hydrologique Centralisateur du Bassin Méditerranéen et l'Agence de Bassin Rhône-Méditerranée-Corse.

La carte $n^{\circ} 1$ indique comment, pour les besoins de l'étude et l'exploitation des enquêtes, les zones inondées de la vallée de l'Orb ont été regroupées en deux " cellules " (Amont» et "Aval " de Tarassac) où les phénomènes hydrologiques sont supposés représentés (quant aux fréquences d'occurence des crues, notamment) par ceux observés aux deux stations de référence correspondantes, Bédarieux et Béziers-Pont Rouge.

Elle indique aussi comment ces cellules sont subdivisées en "secteurs", choisis de façon que les paramètres économiques $y$ soient relativement homogènes (pour faci-

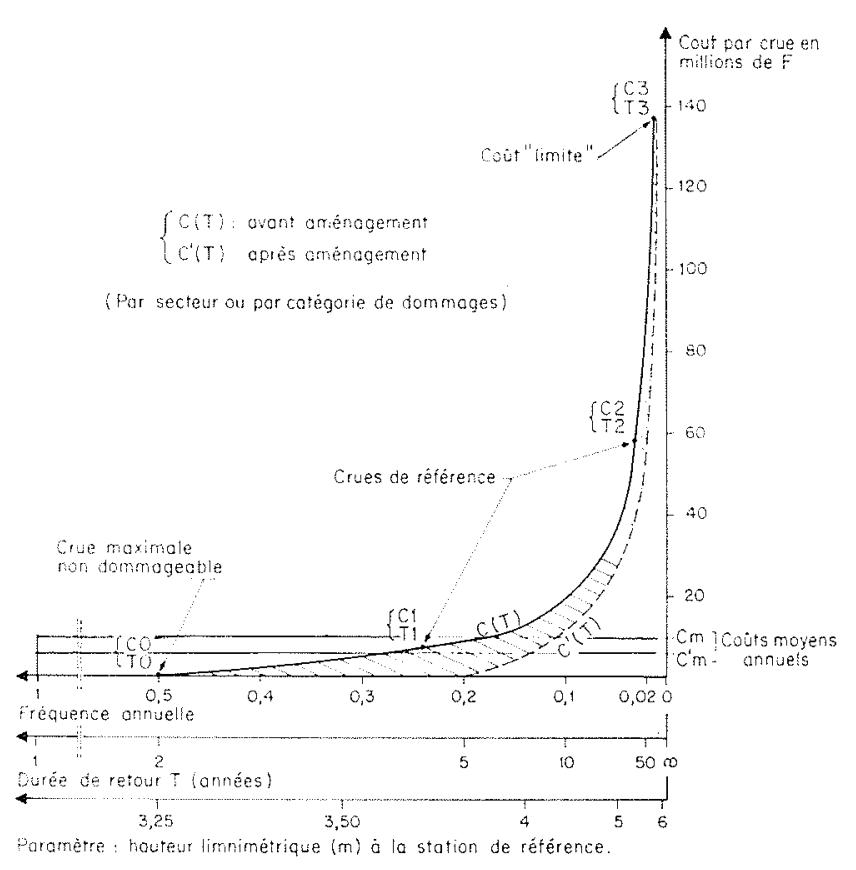

6/ Répartition fréquentielle des coûts avant et après aménagement. Principe.

liter l'exploitation des enquêtes) et aussi de façon à correspondre aux zones d'influence des différents aménagements hydrauliques envisagés. Les courbes fréquentielles de coût des orues ont été établies par secteurs.

\section{Crue de 1969}

Du 18 au 22 octobre 1969, ill s'est produit sur l'Orb une crue assez importante qui, si elle n'a guère affecté le haut bassin du cours d'eau, a causé des inondations notables dans les moyen et bas bassins. On peut estimer que - quant à son débit de pointe $\left(1950 \mathrm{~m}^{3} / \mathrm{s}\right)$ - cette crue correspondait à une durée de retour d'environ 20 ans dans la région de Béziers (2 ans seulement dans le secteur de Bédarieux) (voir figure 2).

Le dispositif qui avait été mis au point pour l'évaluation des dommages dus à une telle crue a fonctionné; l'Institut Géographique National (I.G.N.) et le B.C.E.O.M. sont alors intervenus, pour le compte du Service Hydrologique Centralisateur. Des couvertures photographiques aériennes des zones inondées ont été prises dès que possible $\left(^{c}\right)$ par l'I.G.N., en émulsions panchromatique et infra-rouge; elles ont fait l'objet d'une photo-interprétation qui a permis de délimiter les zones d'inondation $(f)$, de définir les principaux types d'utilisation du sol intéressés et de localiser les dommages. Dans les 'semaines qui ont suivi la crue, les équipes du B.C.E.O.M. ont enquêté sur place de façon à chiffrer avec le maximum de précision les dommages causés dans les différentes zones du bassin, en les répartissant en catégories: dommages mobiliers et immobiliers, artisanaux et commerciaux, industriels, agricoles, dommages aux ouvrages hydrauliques publics et privés, à la voirie, aux autres biens et services publics.

(e) Le 22 octobre, dès que le plafond des nuages se fut suffisamment élevé pour permettre la prise de vue.

(f) Surface d'inondation : environ 5600 ha, dont $60 \%$ de vignobles. 
L'estimation des dommages a été faite par deux types d'enquête: enquête "globale », auprès des mairies et administrations susceptibles d'avoir effectué elles-mêmes des estimations; enquête "analytique", effectuée sur un échantillon de sinistrés convenablement choisi et étendue ensuite à la population totale; l'INSEE (Institut National de la Statistique et des Etudes Economiques) est intervenu pour la détermination des échantillons. Pour cette crue d'octobre 1969, le coût total des dommages sur le cours de l'Orb a ainsi été évalué à $8970000 \mathrm{~F}$, dont plus de la moitié (4870000 F) pour la commune de Béziers. (Le coût total pour l'ensemble du bassin versant serait supérieur de $600000 \mathrm{~F}$ environ).

La crue s'étant produite après la fin des vendanges, les dommages causés à la culture principale, la vigne, s'en sont trouvés réduits. II n'en eût pas été de même si la crue s'était produite plus tôt. On peut alors calculer les dommages agricoles et totaux moyens dus à une crue type 1969 arrivant à une période quelconque de l'année. On arrive au chiffre de $9300000 \mathrm{~F}$ (voir figure 7).

\section{Crue de 1953}

De la même façon. Ie B.C.E.O.M. a recherché, non sans difficulté car beaucoup d'archives ont disparu, la valeur des dommages dus à la crue de décembre 1953, crue beaucoup plus forte que celle d'octobre 1969, et pour laquelle les durées de retour correspondantes peuvent être estimées, pour ce qui concerne les dommages, à 20 à 30 ans pour le secteur de Bédarieux, et 50 à 100 ans pour celui de Béziers (voir figure 2).

Les dommages causés par cette crue sur le cours de l'Orb peuvent être évalués à $4950000000 \mathrm{~F} 1953$, soit $82000000 \mathrm{~F} 1969$ compte tenu de la dépréciation monétaire. Mais une crue telle que celle de 1953, se produisant en 1969, causerait des dommages plus importants que ce dernier chiffre du fait de l'accroissement continu de la valeur économique des biens; les taux annuels de croissance correspondants, variables selon les secteurs économiques et géographiques, ont été évalués, pour la majeure partie des zones inondées, à $2,5 \%$ pour l'agriculture,

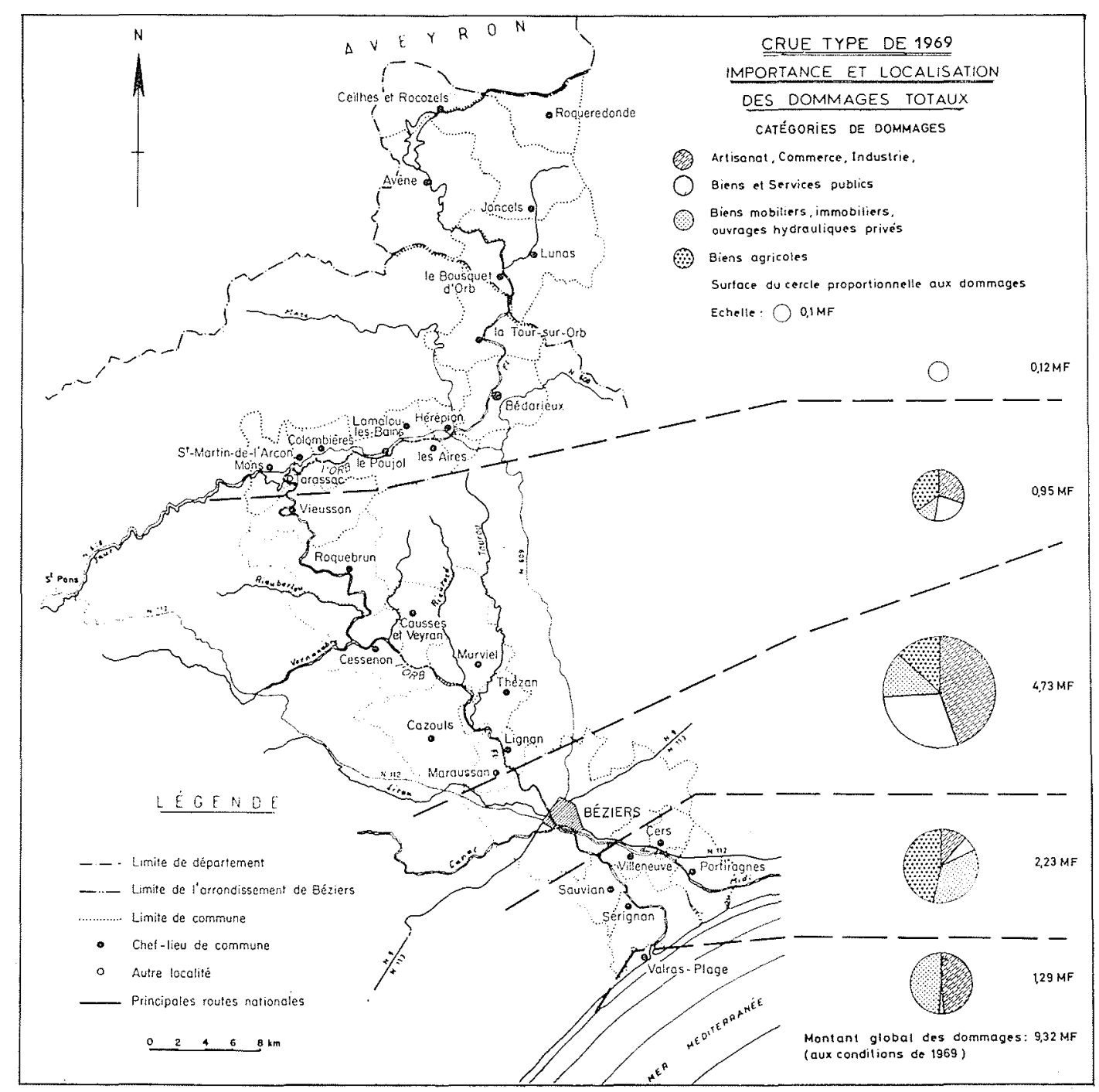


$3 \%$ pour les biens privés, $3,75 \%$ pour l'artisanat, le commerce et l'industrie et $4,5 \%$ pour les biens publics. Il faut encore tenir compte de la correction due à la saison, comme pour la crue de 1969. Au total, la crue type 1953 arrivant en 1969 causerait des dommages évalués à $144000000 \mathrm{~F}$, dont $79000000 \mathrm{~F}$ sur la commune de Béziers (voir figure 8 ).

Pour les deux crues étudiées, on connaît la part des dommages relative à chacun des secteurs en lesquels a été découpée la vallée de l'Orb. Sachant qu'une crue de durée de retour inférieure à 2 ans ne cause pas de dommages sensibles d'inondation (《crue non dommageable»), on peut alors itracer avec assez de précision, au moins pour les secteurs aval, les courbes donnant la répartition fréquentielle des coûts des dommages dus aux crues, dans la situation actuelle. Par planimétrage, on obtient le coût moyen annuel des dommages qui varie de $4900000 \mathrm{~F}$ à $7400000 \mathrm{~F}$ pour l'ensemble des secteurs aval selon que la durée de retour de la crue de 1953 est prise égale à 100 ans ou à 50 ans. Dans les mêmes hypothèses, et si l'on tient compte de l'influence du barrage existant d'Avène sur les crues du haut bassin, le coût moyen total est évalué entre $6400000 \mathrm{~F}$ et $9200000 \mathrm{~F}$.
On peut admettre que, dans ce bassin, te coût moyen annuel des crues va croître, dans le futur, si aucune mesure de protection n'est prise, au rythme des taux de croissance économique constatés dans le passé et déjà mentionnés ci-avant. Les dommages futurs probables peuvent ainsi être évalués pour chaque année à venir, secteur par secteur, et un calcul d'actualisation (au taux de $10 \%$ ) permet allors de calculer leur coût actualisé, que l'on peut rapprocher du coût des ouvrages qui permettraient de les supprimer - ou de les diminuer. Si on appelle $a$ et $i$ les taux d'actualisation et d'évolution économique supposés constants ( $i<a$, en pratique), on voit facilement que le coût actualisé $C$ des dommages futurs s'écrit, en fonction du coût moyen $C_{m}$ :

$$
C=\sum_{1}^{\infty} C_{m} \frac{(1+i)^{n}}{(1+a)^{n}}=C_{m} \cdot \frac{1+a}{a-i}
$$

Dans le cas de l'Orb, le coût total actualisé des crues se situe, dans les mêmes hypothèses que ci-dessus, entre $103 \mathrm{MF}$ et $151 \mathrm{MF}$. Pour des raisons diverses, il a paru prudent d'adopter, pour les calculs d'application, les bornes

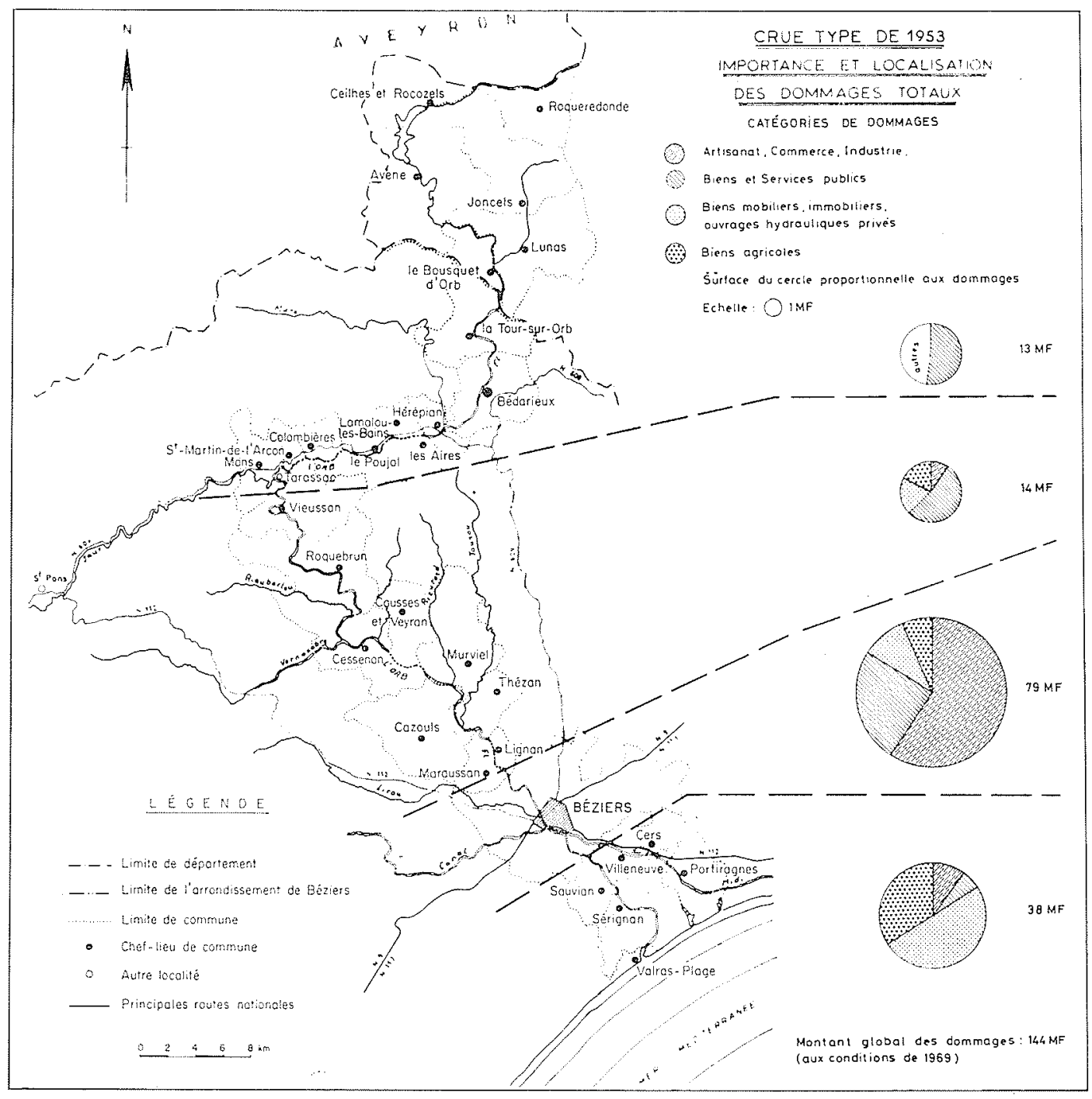


inférieures des intervalles ainsi chiffrés pour les coûts des dommages, soit $6400000 \mathrm{~F}$ pour le coût moyen annuel et $103 \mathrm{MF}$ pour le coût actualisé (dont $22 \mathrm{MF}$ pour la cellule amont de Tarassac et $81 \mathrm{MF}$ pour la cellule aval), ce qui revient à attribuer aux dommages de la crue de 1953 une fréquence centennale.

\section{Les différents aménagements étudiés}

Remarquons que la méthode de calcul économique décrite ci-avant s'applique de la même façon à l'étude de l'incidence d'éventuels aménagements projetés sur le coût des dommages de crues. A une crue type ou naturelle donnée, de probabilité déterminée, l'étude hydraulique doit permettre, en effet, de faire correspondre une crue transformée par l'intervention des ouvrages envisagés, que l'on peut situer dans la famille des crues-types étallonnées en coûts probables de dommages (à partir de la courbe $\mathrm{C}(\mathrm{T})$ de la figure 6), et à laquelle on peut ainsi attribuer une valeur de dommages. D'où une nouvelle courbe de répartition fréquentielle du coût des crues tenant compte de l'existence de l'aménagement et se substituant à celle tracée dans les conditions hydrologiques actuelles (figure 6 , courbe $C^{\prime}(\mathrm{T})$ ). Par différence des deux coûts moyens annuels des dommages de crues - avant et après aménagement - on obtient la recette annuelle (aire hachurée de la fig. 6) procurée par l'aménagement, qui peut être actualisée et comparée au coût des ouvrages pour apprécier l'intérêt économique de leur construction.

Il faut noter alors que l'essentiel des dommages de crues concerne la zone de Béziers et de la basse plaine de l’Orb. On a étudié des aménagements dits "généraux", permettant de protéger au moins l'ensemble de cette zone, et des aménagements plus restreints, ne protégeant que des secteurs localisés. Les uns et les autres sont décrits brièvement ciaprès.

\section{Aménagements généraux}

\section{Canal de dérivation}

avec recalibrage et endiguement du cours d'eau: il s'agit d'un canal de quelque $8 \mathrm{~km}$ de long, contournant Béziers sur la rive droite de l'Orb et permettant de dériver $500 \mathrm{~m}^{3} / \mathrm{s}$ en période de crues, diminuant d'autant le flot traversant la ville. La longueur du canal est limitée au maximum, et ce dernier est complété par un recalibrage de l'Orb même, associé à des endiguements, à l'amont et surtout à l'aval de la dérivation, jusqu’à la mer (voir figure 9).

\section{Recalibrage et endiguements généraux}

Cet aménagement comporte, sur environ $20 \mathrm{~km}$, des travaux de recalibrage du lit, combinés avec des endiguements et des reprises localisées d'ouvrages. Il présente une partie commune avec le précédent, à l'amont et à l'aval de Béziers, mais au canal de dérivation est substitué un recalibrage du lit du cours d'eau dans la ville et un aménagement des obstacles actuels (ponts, seuils d'anciens moulins) (voir figure 9).

On remarquera sur cette figure les ouvrages communs (A et C) aux deux aménagements ci-dessus et les ouvrages propres à chacun d'eux.

\section{Barrage réservoir}

Un barrage-réservoir situé dans la moyenne vallée de l'Orb permettrait d'écrêter les crues à l'aval de son emplacement. Sous réserve d'études plus complètes, certains sites semblent pouvoir être envisagés, en particulier entre Vieus. san et Ceps (voir carte $n^{\circ} 1$ ), et l'un d'eux, celui de Ceps, a été choisi à titre d'exemple pour l'étude (capacité utile de $\left.105 \mathrm{Mm}^{3}\right)$.

\section{Aménagements localisés}

\section{Amélioration de la traversée de Bédarieux}

La ville de Bédarieux, située à l'amont des aménagements précédents, peut être protégée par un recalibrage et des endiguements partiels du cours d'eau, ainsi qu'éventullement grâce à une exploitation du barrage d'Avène - à but agricole - spécialement adaptée.

\section{Ouvrages de protection localisés à Béziers et Valras}

Cette désignation regroupe des travaux de protection intéressant deux des secteurs les plus menacés de la partie aval de l'Orb. Il s'agit de Béziers, où une amélioration pourrait être obtenue par un recalibrage du lit dans la traversée de la ville, associé à un aménagement de certains ouvrages existants (notamment du seuil de Pont-Rouge), et de Valras-Plage, qui pourrait être protégé par une digue contre les flots de l'Orb.

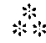

Tous ces aménagements ont fait l'objet d'une étude hydraulique et économique sommaire, permettant de comparer les répartitions fréquentielles des coûts avant et après aménagement pour chaque secteur intéressé. A titre d'exemple, la figure 10 présente les deux courbes correspondantes pour le canal de dérivation et pour le secteur de Béziers. En outre, les principaux résultats ont été regroupés sur le tableau de la figure 11 où sont notamment reportés le coût total des aménagements (y compris frais d'entretien et d'exploitation actualisés), le coût actualisé des dommages de crues avant et après aménagement, le gain actualisé global des opérations envisagées (coûts aux conditions de 1969). A noter que des plus-values foncières non négligeables, dont il n'a pas été tenu compte dans ce tableau, paraîtraient susceptibles d'être obtenues à Béziers et Valras-Plage en cas de protection efficace contre les crues.

Quoique ces chiffres doivent être considérés avec prudence, étant donné le caractère sommaire des études faites, on peut toutefois en condure que la solution du canal de dérivation - qui poserait du reste de difficiles problèmes fonciers - ne paraît pas justifiée sur le plan économique. La solution envisagée d'un recallibrage général du cours d'eau de Béziers à la mer paraît ellle aussi trop coûteuse en regand des résultats à en attendre. La solution du barrageréservoir, qui a l'inconvénient de mobiliser des investissements assez importants, peut paraître à ce stade mériter néanmoins une étude complémentaire, dans l'optique 


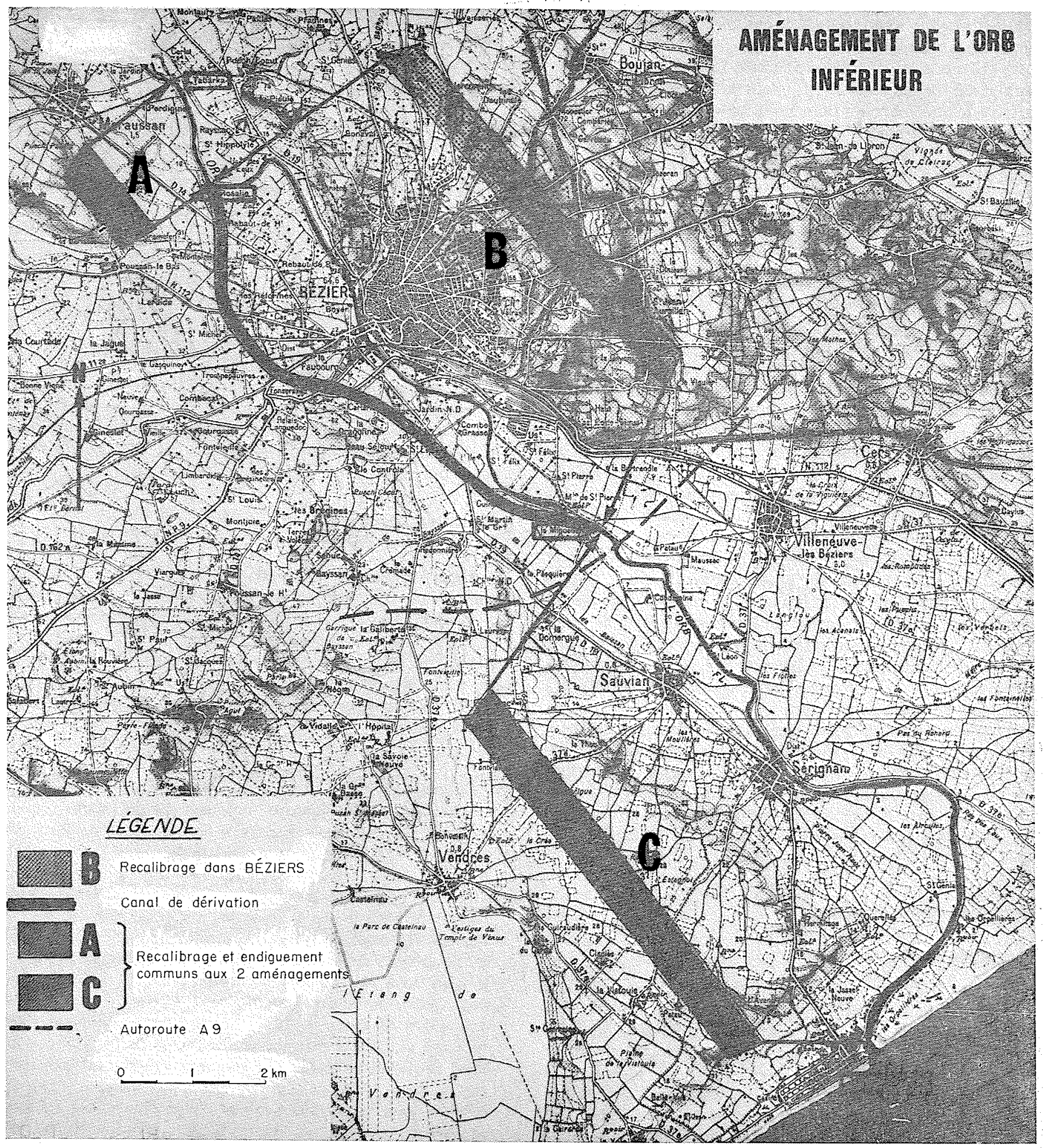




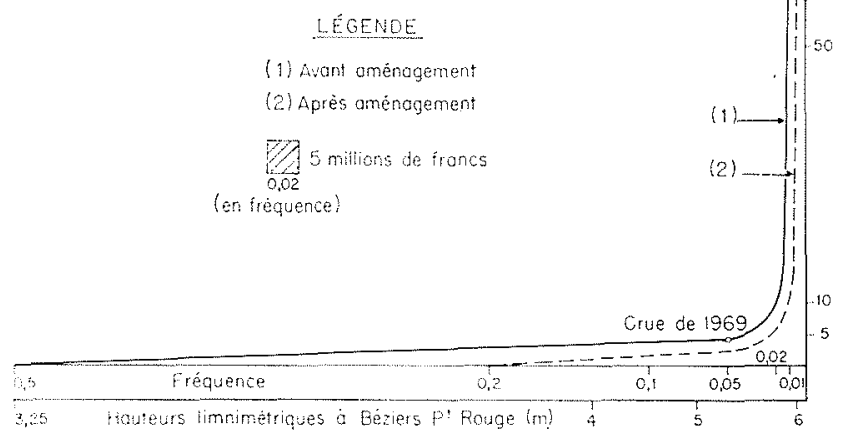

10/ Répartition fréquentielle

des coûts avant et après aménagement

Canal de dérivation : secteur de Béziers.

\begin{tabular}{|c|c|c|c|c|c|c|c|}
\hline $\begin{array}{l}\text { Type } \\
\text { d'aménagement }\end{array}$ & $\begin{array}{c}\text { Coùt des } \\
\text { orrênagements } \\
\text { cn } \\
w \text { de F }\end{array}$ & 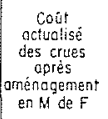 & $\mid \begin{array}{c}\text { Cout } \\
\text { cotol } \\
\text { octualise } \\
\text { en } \\
\text { m de } F\end{array}$ & 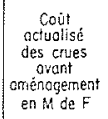 & $\begin{array}{c}\text { Toux de } \\
\text { couverture } \\
\text { en \% }\end{array}$ & $\begin{array}{l}\text { Gain } \\
\text { octuanisé } \\
\text { en } \\
M \text { de } F\end{array}$ & $\begin{array}{c}\text { Gain } \\
\text { relatif } \\
\text { en \% } \%\end{array}$ \\
\hline $\begin{array}{l}\text { Conal de } \\
\text { derivation }\end{array}$ & 94 & 22,7 & 16,7 & 81 & 72 & $-35,7$ & $-38 \%$ \\
\hline $\begin{array}{l}\text { Recoldibroge et } \\
\text { endiguements } \\
\text { geneneraux }\end{array}$ & 74 & 9,4 & 83,4 & 81 & 89 & $-2,4$ & $-3 \%$ \\
\hline $\begin{array}{l}\text { Barrage- } \\
\text { Béservoir }\end{array}$ & 63 & 6,5 & 69,5 & 81 & 92 & $+11,5$ & $+18 \%$ \\
\hline $\begin{array}{l}\text { Ouvroges } \\
\text { local ises }\end{array}$ & 17,8 & 34,3 & 52,1 & 81 & 58 & $+28,9$ & $+162 \%$ \\
\hline (1) & (2) & (3). & $\begin{array}{c}(4)= \\
(2)+(3)\end{array}$ & (5) & $\begin{array}{c}6= \\
(65)-(33) /(5)\end{array}$ & $\begin{array}{c}(7)= \\
(5)-(4)\end{array}$ & $\begin{array}{l}(8)= \\
(7) \neq(2)\end{array}$ \\
\hline
\end{tabular}

11 / Cellule "aval de Tarassac". Compara: on c'es différents aménagements (conditions économiques de 1969).

mentionner les suivantes, à caractère d'utilité du reste général :

- entretien régulier du lit de l'Orb et de ses principaux affluents, ainsi que des ouvrages de protection déjà existants;

- création et utilisation d'un système de prévision des crues;

- enfin aménagement des zones inondables, surtout en secteur urbain. Sur ce dernier point, nous pensons, en effet, que pour de telles zones - qui, quoiqu'on fasse, restent toujours soumises à certains risques d'inondation - des schémas détaillés d'urbanisme, tenant compte de ces risques naturels, gagnent à être mis au point, en vue d'orienter dans un sens convenable le développement ou le remodelage des quartiers construits.
$\mathrm{Au}$ titre des dispositions complémentaires à des aménagements hydrauliques éventuels, il paraît opportun de 


\section{Discussion}

Président : M. BONNEviOT

M. le Président remercie MM. Brun et Dumas pour leur exposé d'un grand intérêt et ouvre la discussion.

M. Banal intervient en ces termes:

L'évaluation du bénéfice à tirer de la protection contre les crues, à partir des courbes donnant le « coût d'une inondation» en fonction de sa probabilité doit, en toute logique, prendre en compte les inondations à venir et non celles du passé; c'est là une cause d'erreur ou plutôt d'incertitude inévitable, mais que l'on peut tenter de réduire.

En ce qui concerne les côts, je voudrais poser deux questions: $1^{\circ}$ Comment est-il tenu compte de l'augmentation systématique de ces derniers avec le développement de l'urbanisation ou de l'industrialisation?

En sens inverse, le perfectionnement des Services d'annonce de crues devrait permettre de réduire certains dégâts en alertant, en temps utile, les intéressés.

$2^{\circ} \mathrm{La}$ durée de la crue a-t-elle une grande influence sur les dommages produits par l'inondation?

M. Brun et M. Srmeon (ce dernier est économiste au B.C.E.O.M. et co-auteur de la méthodologie utilisée par l'Agence financière de Bassin «Rhône-Méditerranée-Corse 》) répondent aux questions cidessus au cours d'un échange de vues qui peut être résumé comme suit :

$1^{\circ}$ Dans l'étude présentée - où il a été effectivement tenu compte de l'évolution future des activités économiques et de la valeur des biens dans la zone submersible - le coût moyen des dommages a été supposé s'accroître chaque année suivant une progression géométrique dont les paramètres ont été estimés d'après le développement prévisible de la région (augmentation de 2,5\% par an pour l'agriculture et de $3,75 \%$ pour l'artisanat, le commerce et l'industrie).
On peut objecter à cette méthode sa tendance à une augmentation exponentielle des coûts (tempérée, il est vrai, par l'actualisation de ces derniers au taux de $10 \%$ par an) $(a)$; on préfère aujourd'hui substituer à ce modèle à croissance exponentielle un modèle à croissance logistique tendant asymptotiquement vers une limite estimée au mieux.

I! ne faut pas oublier d'autre part, de tenir compte du fait que certains biens bénéficient d'une plus-value du seul fait de la construction d'ouvrages de protection contre les inondations, qui peuvent autoriser d'en faire des usages différents et plus lucratifs.

$2^{\circ}$ La durée de la submersion des terrains par la crue infuence fortement — mais à des degrés divers selon les cultures - les dégâts causés en zones agricoles; huit jours de submersion détruisent une plantation de pommiers tandis que la vigne peut résister au moins trois semaines dans les mêmes conditions.

Dans les zones urbaines, l'influence de la durée de la crue est loin d'être nég'igeable; ainsi, dans la région parisienne, lorsque les plus hautes eaux navigables sont dépassées, tout trafic fluvial est arrêté; le dommage causé à la navigation intérieure dépend évidemment de la durée de cet arrêt.

M. le Président clôt à la fois la discussion et la séance de la matinée en se félicitant des fructueux échanges de vues auxquels elle a donné lieu. Ceux-ci peuvent d'ailleurs se poursuivre par correspondance; les contributions qui parviendront, en temps utile, au Secrétariat de la S.H.F. seront intégrées dans le compte rendu de la discussion, joint au texte de la communication qui paraîtra in extenso dans La Houille Blanche.

La séance est levée à 12 heures.

(a) Cette actualisation réduit presque à néant toute augmentation survenant au-delà d'une durée de vingt-cing ans. 